\title{
ON THE SOLUTE DISTRIBUTION AT A MOVING PHASE BOUNDARY*
}

\author{
By K. A. HEIMES (Iowa State University)
}

1. Introduction. Consider an infinite rod of homogeneous binary alloy with a planar solid-liquid interface advancing at constant velocity $R$. Let $D_{i}, v^{i}(x, t)$ denote respectively the diffusion coefficient and solute concentration in the solid $(i=1)$ and liquid $(i=2)$ regions. For $t=0$ we locate the interface at $x=0$ and describe the initial solute compositions by $f_{i}(x)$ for $(-1)^{i} x \geq 0$. In order to obtain equilibrium at the interface, we require

$$
\begin{gathered}
V^{1}(R t, t)=k V^{2}(R t, t), \\
D_{1} V_{x}^{1}(R t, t)-D_{2} V_{x}^{2}(R t, t)=R\left[V^{1}(R t, t)-V^{2}(R t, t)\right],
\end{gathered}
$$

where $k$ is a constant equilibrium distribution coefficient. Assuming no convection in the liquid, the diffusion equations are $D_{i} V_{x x}^{i}=V_{i}^{i}$ in their respective regions. By putting $C^{i}(z, t)=V^{i}(z+R t, t)$ we fix the interface at $z=0$ and move the rod into the solid region $(z<0)$. Consequently, this one-dimensional liquid-solid transformation (solidification) can be described as

Problem S: For $i=1,2$ let $D_{i}, k, R$ be positive constants and let $f_{i}(z)$ be continuous real functions defined for $(-1)^{i} z \geq 0$ with $f_{1}(0)=k f_{2}(0)$. Find functions $C^{i}(z, t)$ for $t \geq 0,(-1)^{i} z \geq 0$ satisfying

$\left(\mathrm{S}_{1}\right) \quad D_{i} C_{z}^{i}+R C_{z}^{i}=C_{t}^{i}$;

$\left(\mathrm{S}_{2}\right) \quad C^{i}(z, 0)=f_{i}(z)$;

$$
C^{1}(0, t)=k C^{2}(0, t), \quad-R(1-k) C^{2}(0, t)=D_{2} C_{z}^{2}(0, t)-D_{1} C_{z}^{1}(0, t) .
$$

The corresponding solid-liqiud transformation (Problem M for melting) is the same as Problem $\mathrm{S}$ except that $R$ is replaced by $-R$ in $\left(\mathrm{S}_{1}\right)$ and $\left(\mathrm{S}_{3}\right)$. Problem $\mathrm{S}$ has been solved for cases $D_{1}=D_{2}$ and $D_{1}=0$ with particular initial conditions in [1] and [2].

In this paper we give sufficient conditions that both the above problems have unique solutions and explicit solutions are obtained by Laplace transforms methods. Both problems reduce to solving an integral-differential for the function $g(t)=C^{1}(0, t)=$ $k C^{2}(0, t)$ which describes the time behavior of solutions at the interface.

2. The reduced problem. We first show that problems $M$ and $S$ are equivalent to

Problem A: Given positive constants $K, \lambda$ and real functions $g_{i}(x)$ for $x \geq 0$ with $g_{1}(0)=g_{2}(0)$, find $u^{i}(x, y)$ for $x \geq 0, y \geq 0$ so that

$$
\left(\mathrm{A}_{1}\right) \quad u_{x x}^{i}=u_{y}^{i}
$$

* Received December 30, 1970; revised version received April 12, 1971. Work performed in part at Ames Laboratory, U. S. Atomic Energy Commission. Contribution No. 2911. 
(A $\left.\mathrm{A}_{2}\right) \quad u^{i}(x, 0)=g_{i}(x)$;

$\left(\mathrm{A}_{3}\right) \quad e^{-y} u^{2}(0, y)=e^{-\lambda y} u^{1}(0, \lambda y), \quad(1-K) e^{-y} u^{2}(0, y)=e^{-y} u_{x}^{2}(0, y)+K e^{-\lambda y} u_{x}^{1}(0, \lambda y)$.

Functions $u^{i}(x, y)$ will be called solutions to problem $A$ when (i) for $x \geq 0, y \geq 0$ they are continuous, satisfy $\left(A_{2}\right)$ and estimates of the form $|u(x, y)|<M e^{m x^{2}}$ on compact $y$ intervals; (ii) for $x>0, y>0 u_{x x}^{i}$ and $u_{y}^{i}$ are continuous and satisfy $\left(\mathrm{A}_{1}\right)$; (iii) for $x \geq 0, y>0 u_{x}^{i}$ is continuous and $\left(\mathrm{A}_{3}\right)$ holds.

Straightforward calculations verify

Lemma 1. Let $u^{1}, u^{2}$ solve problem A with $K=k, \lambda=D_{2} / D_{1}$ and $g_{1}(\lambda x)=e^{\lambda x} f_{1}(-z)$, $g_{2}(x)=K e^{-x} f_{2}(z)$ where $x=R z / 2 D_{2}, y=R^{2} t / 4 D_{2}$. Then

$$
\begin{aligned}
& C^{1}(z, t)=e^{\lambda(x-y)} u^{1}(-\lambda x, \lambda y) \\
& C^{2}(z, t)=\frac{1}{K} e^{x-y} u^{2}(x, y)
\end{aligned}
$$

solve problem M. Similarly, if $u^{1}, u^{2}$ solve problem A for $K=1 / k, \lambda=D_{1} / D_{2}$ and $g_{1}(\lambda x)=e^{\lambda x} f_{2}(-z), g_{2}(x)=K e^{-x} f_{1}(z)$ where $x=-R z / 2 D_{1}, y=R^{2} t / 4 D_{1}$, then

$$
\begin{aligned}
C^{1}(z, t) & =\frac{1}{K} e^{x-y} u^{2}(x, y) \\
C^{2}(z, t) & =e^{\lambda(x-y)} u^{1}(-\lambda x, \lambda y)
\end{aligned}
$$

solve problem S.

LEMma 2. For $p, q \in C[0, \infty)$, solutions to $u_{x \varepsilon}=u_{y}$ for $x>0, y>0$ with $u(x, 0)=$ $p(x), u(0, y)=q(y)$ are unique.

Proof: This is a standard application of the maximum principle for the heat equation. Details are similar to those given on p. 48 of [3].

Define the functions

$$
\begin{aligned}
S(x, y) & =(4 \pi y)^{-1 / 2} \exp \left(-x^{2} / 4 y\right) & & \\
U^{i}(x, y) & =g_{i}(x), & & y=0, \\
& =\int_{0}^{\infty}[S(x-r, y)+S(x+r, y)] g_{i}(r) d r, & & y>0 .
\end{aligned}
$$

Then the $U^{i}$ solve $\left(A_{1}\right),\left(A_{2}\right)$ with $U_{x}^{i}(0, y)=0$ and

$$
U^{i}(0, y)=2 \int_{0}^{\infty} S(r, y) g_{i}(r) d r=\frac{2}{\sqrt{ } \pi} \int_{0}^{\infty} e^{-\varkappa^{*}} g_{i}(2 u \sqrt{ } y) d u
$$

(see p. 53 of [3]).

Suppose that $u^{1}, u^{2}$ solve Problem A with $g_{1}, g_{2}, g \in C[0, \infty) \cap C^{\prime}(0, \infty)$ where $g(y)=e^{-y} u^{2}(0, y)=e^{-\lambda y} u^{1}(0, \lambda y)$ is the interface function. It then follows from Lemma 2 that

$$
\begin{aligned}
& u^{1}(x, y)=U^{1}(x, y)+\int_{0}^{\nu}\left(\operatorname{erfc} \frac{x}{2(y-r)^{1 / 2}}\right) \frac{d}{d r}\left[e^{r} g(\mu r)-U^{1}(0, r)\right] d r \\
& u^{2}(x, y)=U^{2}(x, y)+\int_{0}^{\nu}\left(\operatorname{erfc} \frac{x}{2(y-r)^{1 / 2}}\right) \frac{d}{d r}\left[e^{r} g(r)-U^{2}(0, r)\right] d r
\end{aligned}
$$


where $\mu=1 / \lambda$. That is, the right-hand sides in (5) solve $\left(\mathrm{A}_{1}\right)$ and agree with $u^{i}(0, y)$ and $u^{i}(x, 0)$. Substituting (5) in the last equation of condition $\left(\mathrm{A}_{3}\right)$, we see that $g(y)$ solves

$$
\begin{aligned}
(1-K) g(y)=e^{-y} \int_{0}^{\nu}[\pi(y & -r)]^{-1 / 2} \frac{d}{d r}\left[U^{2}(0, r)-e^{r} g(r)\right] d r \\
& +K e^{-\lambda y} \int_{0}^{\lambda y}[\pi(\lambda y-r)]^{-1 / 2} \frac{d}{d r}\left[U^{1}(0, r)-e^{r} g(\mu r)\right] d r .
\end{aligned}
$$

These steps are reversible, giving

Lemma 3. Let $W=C[0, \infty) \cap C^{\prime}(0, \infty)$. For initial conditions $g_{i} \in W$, Problem A has solutions $u^{i}$ with $u^{i}(0, y) \in W$ if and only if there exists a function $g \in W$ solving (6) with $g(0)=g_{i}(0)$.

For the trivial case $K=\lambda=1$ we see that $g(y)=\left(e^{-\nu} / 2\right)\left[U^{1}(0, y)+U^{2}(0, y)\right]$.

Denote the transform of a function by a superscript *. All the formulas used below may be found in [4].

From (4) we have $U^{i *}(0, s)=g_{i}^{*}(\sqrt{ } s) / \sqrt{ } s$. Transforming (6) and solving for $g^{*}$, we obtain

$$
g^{*}(s)=D^{*}(s)\left\{K_{\mu} g_{1}^{*}\left((\mu s+1)^{1 / 2}\right)+g_{2}^{*}\left((s+1)^{1 / 2}\right)\right\},
$$

where $D^{*}(s)=\left[\left((s+1)^{1 / 2}+1\right)+K\left((\mu s+1)^{1 / 2}-1\right)\right]^{-1}$ and $\mu=1 / \lambda$. Except for the easy case $K=\lambda=1$ we can write

$$
D^{*}(s)=\frac{\left((s+1)^{1 / 2}+1\right)-K\left((\mu s+1)^{1 / 2}+1\right)}{\left((s+1)^{1 / 2}+1\right)\left(A(s+1)^{1 / 2}+B\right)},
$$

where $A=1-K^{2} \mu$ and $B=1-2 K+K^{2} \mu$. Given specific initial conditions $g_{1}, g_{2}$, one would now simplify (7) using (8) prior to inversion. In general we get the convolution

$$
g(y)=\frac{2}{\sqrt{ } \pi} \int_{0}^{\nu} d t \int_{0}^{\infty} r \sqrt{ } t e^{-r^{\prime} t} D(y-t)\left[e^{-t} g_{2}(2 r t)+K \sqrt{ } \mu e^{-\lambda t} g_{1}(2 \sqrt{ } \lambda r t)\right] d r,
$$

where

$$
\begin{aligned}
& D(y)=\frac{G(y)}{1+K \sqrt{ } \mu}+\frac{(\sqrt{ } \mu-1)}{2(K \mu-1)}[F(y)-G(y)]+\frac{\sqrt{ } \mu}{2(K \mu-1)} \int_{0}^{y}\left[F(t)-G^{\prime}(t)\right] E(y-t) d t, \\
& E(y)=\left[e^{-\lambda y}-e^{-y}\right] /\left(4 \pi y^{3}\right)^{1 / 2}, \quad F(y)=\left(e^{-y} /(\pi y)^{1 / 2}\right)-\operatorname{erfc} \sqrt{ } y, \\
& G(y)=0 \\
& K^{2}=\lambda \\
& =\left(e^{-\nu} /(\pi y)^{1 / 2}\right)-Q \exp \left(\left(Q^{2}-1\right) y\right) \operatorname{erfc} Q \sqrt{ } y \quad K^{2} \neq \lambda, Q=B / A .
\end{aligned}
$$

When $K_{\mu}=1$ we have $Q=1$ and $F=G$ so the correct formula for $D$ is obtained by computing $\lim _{K_{\mu \rightarrow 1}}[F(y)-G(y)] /\left(K_{\mu}-1\right)$.

From Eq. (9) it follows that if the initial conditions $g_{1}, g_{2}$ belong to the class of functions $W$ defined in Lemma 3 and have Laplace transforms, the same is true for $g$. The function $g(y)$ in (9) then solves Eq. (6) and provides solutions, via (5), to Problem A. Uniqueness of such solutions is clear from Eq. (7). Thus we have

Theorem: For initial conditions $g_{i}(x)$ in class $W$ with $\left|g_{i}(x)\right| \leq M_{i} \exp \left(m_{i} x\right)$ for large $x$, Problem $\mathrm{A}$ has a unique solution with interface function satisfying the same conditions. The solution is given by (5) for $g(y)$ in (9). 
The formulas in (5) and (9) are less than elegant. However, for any particular problem one can usually perform some additional simplifications, transform (5) back to problem M or S using (1) or (2) and study the solutions numerically. Approximations are also easily accessible. For example, one can choose a simple approximation for $g(y)$ in (9), select one of the initial conditions $g_{1}$ or $g_{2}$ and solve for the remaining function $\left(g_{2}\right.$ or $\left.g_{1}\right)$ using (7). Then Eq. (5) gives solutions to problem A except that one of the initial conditions differs from the original. If the difference is small, an approximate solution is obtained.

A study of problem $M$ for specific initial conditions using the above procedure is given in [5].

\section{References}

[1] W. A. Tiller and R. F. Sekerka, Redistribution of solute during phase transformation, J. Appl. Phys. 33, 2726 (1964)

[2] V. G. Smith, W. A. Tiller and J. W. Rutter, A mathematical analysis of solute redistribution during solidification, Canad. J. Phys. 33, 723 (1955)

[3] G. Hellwig, Partial differential equations, Blaisdell, 1964

[4] G. E. Roberts and H. Kaufman, Table of Laplace transforms, W. B. Saunders, 1966

[5] J. D. Verhoeven and K. A. Heimes, The initial transient in melting and solidification experiments. Int. J. Crystal Growth (to appear) 